\title{
Optical Phase Transitions in Photonic Networks: a Spin-System Formulation
}

\author{
Alba Ramos®, Lucas Fernández-Alcázar, and Tsampikos Kottos๑* \\ Wave Transport in Complex Systems Lab, Department of Physics, \\ Wesleyan University, Middletown, Connecticut 06459, USA \\ Boris Shapiro \\ Technion-Israel Institute of Technology, Technion City, Haifa 32000, Israel
}

(Received 13 December 2019; revised 5 April 2020; accepted 1 June 2020; published 31 July 2020)

\begin{abstract}
We investigate the collective dynamics of nonlinearly interacting modes in multimode photonic settings. To this end, we have established a connection with the theory of spin networks. The emerging "photonic spins" are complex, soft (their size is not fixed), and their dynamics has two constants of motion. Our analysis sheds light on the nature of the thermal equilibrium states and reveals the existence of optical phase transitions which resemble a paramagnetic to a ferromagnetic and to a spin-glass phase transition occurring in spin networks. We show that, for fixed optical power, these transitions are driven by the type of the network connectivity, its coordination number, and the total energy of the optical signal. In strictly onedimensional photonic networks we establish a universal one-parameter scaling that dictates the crossover from a (quasi)ferromagnetic phase to a paramagnetic phase.
\end{abstract}

DOI: $10.1103 /$ PhysRevX.10.031024

\section{INTRODUCTION}

In physics one often encounters problems involving a great number of nonlinear interacting modes. Such problems naturally arise in statistical mechanics [1-3], hydrodynamics [4,5], matter waves [6-8], and more. An emerging framework is in photonics, where light propagation in nonlinear multimode optical structures have recently attracted a lot of attention [9-19]. Mixing between linear modes, due to nonlinearity, results in redistribution of the energy among the modes. Understanding of this process is important both theoretically and for possible applications. Brute-force computations for this purpose are either impossible (due to the large number of degrees of freedom involved) or unsatisfactory as far as the understanding of the underlying physics that dictates the energy redistribution. Such understanding might contribute to a design of more efficient high-power light sources [17], highresolution imaging schemes [20-22], and high-speed telecommunication systems [23-25].

In this regard, a number of recent papers have promoted well-established equilibrium $[9,11,19,26-28]$ and nonequilibrium [15,29-32] thermodynamics techniques as a viable

\footnotetext{
*tkottos@wesleyan.edu
}

Published by the American Physical Society under the terms of the Creative Commons Attribution 4.0 International license. Further distribution of this work must maintain attribution to the author(s) and the published article's title, journal citation, and DOI.
Subject Areas: Nonlinear Dynamics,

Photonics, Statistical Physics theoretical toolkit which can be used for better understanding of nonlinear multimode optical systems. In particular, the authors of Refs. [19,26], assuming thermal equilibrium conditions and weak nonlinearity, have established a comprehensive optical thermodynamics formalism allowing us to design potentially novel classes of highpower multimode optical structures or efficient cooling schemes. For weak nonlinearity, the specific nature of the nonlinear mode interaction (e.g., Kerr or saturable or thermal nonlinearities) is irrelevant. Its role is important for the thermalization process but not for the properties of the equilibrium state.

Of course, the question of thermalization in a given isolated optical system is a subtle issue. In general, it can only be answered $a$ posteriori by observing the long-time dynamics of the system prepared in some initial nonequilibrium state. Furthermore, by "thermalization" here we always mean that the optical system reaches an internal equilibrium, with temperature which generally has nothing to do with that of the environment, i.e., with the walls of the resonators or with the optical fiber. Although there are indications that such quasiequilibrium states can be reached in optical systems (e.g., see the phenomenon of the beam self-cleaning [18,33-37]), the overall situation is far from being clear, attracting currently a lively debate between researchers. Below, the feasibility of thermalization will remain the main assumption of our analysis.

The implementation of statistical thermodynamics methods in modern photonics opens up a new arena where ideas and concepts from statistical mechanics can be transferred 


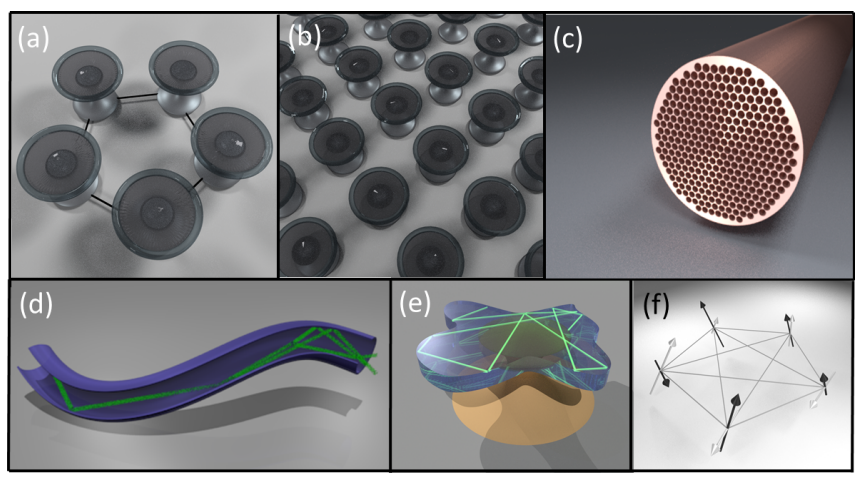

FIG. 1. A variety of photonic nonlinear multimode networks, whose field dynamics is modeled by an effective coupled mode theory of the type given by Eqs. (1) and (2). (a) A onedimensional and (b) two-dimensional photonic lattice consisting of coupled microresonators. (c) A multicore fiber. (d) A multimode fiber. (e) A deformed multimode (micro)resonator with underlying chaotic dynamics. (f) A network of coupled "soft" (size-modulated) spins. The corresponding Hamiltonian has similarities but also crucial differences with the Hamiltonian that describes a photonic multimode network.

to optics and utilized for light control. A prominent example is the notion of phase transitions characterized by changes in the properties of a system as an external parameter varies [2]. Maybe the most celebrated area of physics where such phenomena have been extensively studied is the theory of spin-network models [38-40]. These studies indicated that the phase transition can be understood as a competition between the interactions among spins, which facilitates order, and the thermal fluctuations (the entropy) causing random disturbances. In many occasions, these phase transitions are associated with symmetry breaking phenomena. To understand them, one has to analyze the nature of the thermal equilibrium state of the interacting system. In this paper, we show that there are certain analogies between the statistical thermodynamics of spin models and multimode photonic networks. Inspired by these analogies, we ask questions such as the following. Are the phases of the electromagnetic field correlated or are they entirely random? Is the optical power distributed more or less evenly between all modes of the entire system, or can some finite fraction of the total optical power reside in a single mode? How do the topology of the intermode connectivity and the randomness in the coupling affect the nature of the thermal state?

It turns out that these questions can be related. In previous works, for example, it was shown that macroscopic occupation of a single photonic mode does occur and, moreover, it can happen even in linear systems (we stress again that weak nonlinearity can be neglected only in the equilibrium state, while it is crucial in the thermalization process) [27,29]. It is quite remarkable, thus, that a purely classical system exhibits a phenomenon like BoseEinstein condensate (BEC) transition in a quantum Bose gas. Actually, there have been a number of experiments demonstrating that, in the course of propagation along the fiber, optical power "flows" toward the lower modes [18,33-37]. The origin of this beam "self-cleaning" remains an open issue with various research groups offering a variety of different tentative explanations. Potential scenarios involve the importance of parametric instabilities that lead to a spatial mode redistribution process [36], or the importance of weak (propagation-dependent) disorder [37,41] and chaotic cross section [19] that dramatically accelerates the rate of thermalization and condensation.

In this paper, we demonstrate that the connectivity of an optical structure is an important factor in its thermalization process: it affects the type of the optical phase transitions and the nature of the thermal equilibrium state. The question is not only of fundamental importance, it pertains also to recent photonic developments where networks with complex connectivities can be realized; see Figs. 1(a)-1(e). Specifically, we show that in the case of long-range couplings, the nonlinearity is instrumental for achieving optical phase transitions. In fact, by identifying an order parameter that is equivalent to the magnetization in spin-network models, we are able to show both theoretically and numerically the existence of a ferromagneticto-paramagnetic phase transition in long-range coupling networks, analogous to the one occurring in spin systems. Furthermore, we show that the theoretical description of this phase transition can be achieved by invoking a meanfield approach which is qualitatively applicable for a broader range of photonic lattices, including those with nearest-neighbor couplings. For strictly one-dimensional photonic networks we established a universal oneparameter scaling theory that describes their crossover from "quasiferromagnetic" state to a paramagnetic state. Finally, when disorder is introduced into the couplings of long-range connectivity networks, the system might undergo another type of a transition, namely to a spinglass phase, much as in the case of frustrated coupled spins.

Although these analogies between photonic multimode networks [Figs. 1(a)-1(e)] and spin networks [Fig. 1(f)] is useful, one needs to keep in mind that the two problems have important differences. Specifically, our "photonic spins" are complex dynamical variables (amplitudes of the electric field). Moreover, they fluctuate not only in their direction but also in their size. We expect that the analogies drawn from our study will bring together two seemingly different areas: statistical mechanics of spin networks and light transport in nonlinear multimode settings. This cross-fertilization will, hopefully, allow the development of better design strategies for the control of light transport in multimode photonic networks.

It is instructive to point out that similar analogies between photonic networks and spin systems have also appeared in the framework of active optical multimode configurations, with gain and loss, under the condition of 
constant pumping [42-47]. Such systems, in contrast to our study, are far from equilibrium and the whole idea of a thermodynamic description is not applicable. While in many cases terminology borrowed from equilibrium statistical mechanics (like ferromagnetic or paramagnetic phases) has been used in some of these references, these terms have a completely different meaning from that in equilibrium statistical mechanics (e.g., the "ferromagnet" is associated with the, essentially nonequilibrium, phenomenon of mode locking). Also, since their system has loss and gain, the generated dynamics is different from ours and does not conserve energy and norm.

The structure of this paper is as follows. In Sec. II, we discuss the general statistical thermodynamics formalism associated with the analysis of optical thermal equilibrium states. Special attention is given to the case of weak nonlinearities where we derive the occupation number statistics. In Sec. III, we analyze a class of complex multimode photonic networks with long-range connectivity. Two cases are discussed in detail: the case of constant couplings and the opposite case of random couplings. We show that under specific conditions these systems demonstrate optical phase transitions from ferromagnetic to paramagnetic and spin-glass phases. Photonic networks with constant nearest-neighbor couplings are analyzed in Sec. IV using a mean-field formalism and a scaling theory (for strictly one-dimensional networks). Our conclusions are summarized in Sec. V.

\section{GENERAL FORMALISM}

\section{A. Theoretical modeling of photonic networks}

The dynamics of nonlinear multimode photonic networks in Fig. 1 can be modeled using the framework of time-dependent coupled mode theory. The associated equations are

$$
i \frac{d \psi_{l}}{d t}=-\sum_{j} J_{l j} \psi_{j}+\chi\left|\psi_{l}\right|^{2} \psi_{l}, \quad l=1, \ldots, N,
$$

where $\psi_{l}$ is the degree of freedom (the complex amplitude) at node $l$ of the network and $J_{l j}=J_{j l}^{*}$ is the connectivity matrix that dictates the couplings among the nodes. We will typically assume zero self-coupling terms $J_{l l}=0$. Finally, the last term in Eq. (1) describes the nonlinearity due, for instance, to Kerr effect.

On a formal and general level $\psi_{l}=\langle l \mid \psi\rangle$ are components of the electric field (with some fixed polarization) in some basis of orthonormal modes $\{|l\rangle\}$ (the "basic modes"). The choice of the set of these modes depends on the problem at hand. For instance, for the case of coupled single-mode microresonators [48] [Figs. 1(a) and 1(b)] the index $l$ labels the resonators, and the basic mode $|l\rangle$ is the eigenmode of the $l$ th resonator, decoupled from the rest of the network (we treat the resonators as structureless point objects). In this framework, the coefficients $J_{l j}=J_{j l}^{*}$ (for $l \neq j$ ) represent evanescent couplings between different resonators and $J_{l l}=\omega_{l}$ is the eigenfrequency of resonator $l$ (with nonlinearity neglected), which in case of identical resonators can be set to be zero, i.e., $\omega_{l}=0(l=1, \ldots, N)$. The same interpretation applies to a multicore fiber [25], Fig. 1(c), where now $l$ labels the single-mode fibers. In Fig. 1(c) the propagation direction $z$ plays the role of time.

It is important to clearly distinguish between the basic modes, like an eigenmode of an isolated resonator in Fig. 1(a), and the eigenmodes of the entire structure, i.e., the stationary solutions of the entire system of coupled equations (1) (with the nonlinearity neglected). The latter are often referred to as "supermodes." For instance, when we are talking about condensation of the optical power in a single mode, we mean of course the supermode (with the lowest energy) and not an eigenmode of a single resonator. Sometimes, where no confusion can arise, we will use the term "mode" instead of "supermode." The supermodes $f_{\alpha}(l)$ form a complete basis, so the field $\psi_{l}(t)$ can be expanded as $\psi_{l}(t)=\sum_{\alpha} C_{\alpha}(t) f_{\alpha}(l)$, reducing Eq. (1) to

$$
i \frac{d C_{\alpha}}{d t}=\varepsilon_{\alpha} C_{\alpha}(t)+\chi \sum_{\beta \gamma \delta} \Gamma_{\alpha \beta \gamma \delta} C_{\beta}^{*}(t) C_{\gamma}(t) C_{\delta}(t),
$$

with

$$
\Gamma_{\alpha \beta \gamma \delta}=\sum_{l} f_{\alpha}^{*}(l) f_{\beta}^{*}(l) f_{\gamma}(l) f_{\delta}(l) .
$$

As we show below, this mode representation of the evolution Eq. (1) is particularly useful when the nonlinearity is weak, and it often appears in the description of various optical settings [49,50].

Equation (2) also describes multimode optical fibers [25,51-53] or multimode resonators [53-57]; see Figs. 1(d) and $1(\mathrm{e})$, respectively. In this case the supermodes are the eigenmodes of the multimode fiber or cavity in the absence of nonlinearities, and unlike the previous case, when the basic modes were localized in space (on a single resonator), now they extend over the entire structure. The $\epsilon_{\alpha}$ describe the corresponding propagation constants or resonant frequencies. When Eq. (2) models a multimode fiber, the variable $t$ plays the role of propagation distance.

In our modeling Eqs. (1) and (2) the coupling matrix $J$ does not depend on time (or propagation distance in case of fibers). Of course, this is appropriate for arrays of coupled resonators [Figs. 1(a) and 1(b)] and chaotic multimode resonators [Fig. 1(e)]. On the other hand, a more appropriate modeling of light propagation in deformed multimode fibers [Fig. 1(d)] requires us to take into consideration the fact that the coupling matrix $J$ varies over large propagation distances ("dynamical" disorder). Our study, however, is concentrated on the equilibrium 
properties of the system (specifically the existence of phase transitions) and we do not analyze thermalization dynamics in detail. Equilibrium is reached either for constant or for propagation-dependent coupling matrices $J$. In the former case it requires longer thermalization lengths which might not be realistic when compared with experiments. Recent numerical calculations [19], however, indicated that the thermalization length might be considerably shortened in case of fibers with chaotic (quenched) cross section. At the same time, it was found that weak dynamical disorder dramatically enhances the thermalization process toward thermal equilibrium $[37,41]$.

The equation of motion (1) is derivable from the energy functional (the Hamiltonian):

$$
\mathcal{H}\left\{\psi_{l}(t)\right\}=-\sum_{l, j} J_{l, j} \psi_{l}^{*} \psi_{j}+\frac{1}{2} \chi \sum_{l}\left|\psi_{l}\right|^{4} \equiv E
$$

In the course of time the total energy $E$ and the total optical power,

$$
\mathcal{N}\left\{\psi_{l}(t)\right\}=\sum_{l}\left|\psi_{l}(t)\right|^{2} \equiv A,
$$

are conserved. Our modeling ensures that both the total power $A$ and the energy $E$ are extensive quantities, proportional to the number of modes $N$, as appropriate for thermodynamics.

\section{B. Problem of thermalization}

An important question is whether an isolated system of interacting modes eventually thermalizes, i.e., reaches an equilibrium state which can be described by just two parameters - the inverse temperature $\beta$ and the chemical potential $\mu$, which in turn are determined by the energy $E$ and the total power $A$ of the initial preparation. If such an equilibrium state is reached, the system can be analyzed using the well-established methods of statistical mechanics and thermodynamics.

For example, a statistical mechanics description of the system of Eq. (1) is achieved by calculating the classical grand-canonical partition function $\mathcal{Z}$,

$$
\mathcal{Z}=\int\left(\prod_{l=1}^{N} d \psi_{l}^{*} d \psi_{l}\right) e^{-\beta \mathcal{H}+\beta \mu \mathcal{N}},
$$

where the Lagrange multipliers $\beta=1 / T$ and $\mu$ have been introduced (in analogy with the inverse temperature and the chemical potential) to ensure conservation (on average) of $E$ and $A$, respectively [see Eqs. (4) and (5)]. Specifically, the relation between the microcanonical quantities,

$$
a \equiv \frac{A}{N}, \quad h \equiv \frac{E}{N},
$$

which describe the average optical power $a$ and averaged energy density $h$ per mode and the grant canonical quantities $\mu, \beta$, is given by

$$
a=\frac{1}{\beta N} \frac{\partial \ln (\mathcal{Z})}{\partial \mu}, \quad h=-\frac{1}{N} \frac{\partial \ln (\mathcal{Z})}{\partial \beta}+\frac{\mu}{N \beta} \frac{\partial \ln (\mathcal{Z})}{\partial \mu} .
$$

Using the partition function as a starting point, we can next calculate the thermodynamic potential,

$$
\Omega=-T \ln (\mathcal{Z}),
$$

and from there, the entire "optical thermodynamics" can be derived. For instance, the entropy is $S=-(\partial \Omega / \partial T)_{\mu}$.

We note that the problem of thermalization in nonlinear lattices, under time evolution defined in Eq. (1) (primary in one spatial dimension and with $J_{l j}$ restricted to nearest neighbors only), has also been addressed in the framework of statistical mechanics [58-61]. In these studies, it has been pointed out that thermalization with positive temperature occurs only in a certain region of the $(E, A)$ plane. For fixed total optical power $A$, this occurs only if the energy is not too large [58]. The maximum value $h=h_{\max }$ for a given average norm $a$ per site corresponds to $\beta \rightarrow 0$ (infinite temperature) and $\mu \rightarrow-\infty$, and it is

$$
h_{\max }=\chi a^{2} .
$$

For larger energies, in some cases, the system acquires a Gibbs distribution with negative temperature, as shown in Refs. [26,58]. In this case we have an inverted population scenario where more power populates the higher-energy levels. Finally, for sufficiently large $E$ the norm cannot spread uniformly over the entire system and high-amplitude peaks of $\psi_{l}$ (breathers [62]) emerge. Below, we confine our analysis to the domain where the temperature is positive.

\section{Thermal equilibrium in the case of weak nonlinearities}

In many optics applications the nonlinearity is considered weak. It is of course essential in the mode-mixing process [see Eq. (2)] needed for reaching equilibrium. The total energy and power, however, are dominated by the linear term in the Hamiltonian, i.e., to a good approximation,

$$
E=\sum_{\alpha=1}^{N} \varepsilon_{\alpha}\left|C_{\alpha}\right|^{2}, \quad A=\sum_{\alpha=1}^{N}\left|C_{\alpha}\right|^{2} .
$$

Here $\left|C_{\alpha}\right|^{2}$ is the (normalized) optical power in mode $\alpha$. Using these expressions Christodoulides and co-workers [19,26] were able to develop a kind of "optical thermodynamics," identifying the optical analogy of entropy, equation of states, and other quantities. Below, we briefly summarize this theory, using the grand-canonical formulation Eq. (6) discussed above. 
Assuming that the system has thermalized, its grand partition function, nonlinearity being neglected, is given by the product of independent modes contributions,

$\mathcal{Z}=\prod_{\alpha=1}^{N}\left[\int d C_{\alpha}^{*} d C_{\alpha} e^{-\beta\left(\varepsilon_{\alpha}-\mu\right)\left|C_{\alpha}\right|^{2}}\right]=\prod_{\alpha=1}^{N}\left[\frac{\pi}{\beta\left(\varepsilon_{\alpha}-\mu\right)}\right]$,

where $\beta=1 / T$ and $\mu$ can be found from the constraints in Eq. (11). It immediately follows from Eq. (12) that the average of optical power in mode $\alpha$ obeys the RayleighJeans distribution [19,26,27,29-31] (see also Ref. [63]):

$$
\left\langle\left|C_{\alpha}\right|^{2}\right\rangle=\frac{T}{\varepsilon_{\alpha}-\mu} \equiv \bar{n}_{\alpha} .
$$

From Eq. (13) we can calculate the thermodynamic potential Eq. (9) which takes the form

$$
\Omega=-T \sum_{\alpha=1}^{N} \ln \left(\frac{\pi T}{\varepsilon_{\alpha}-\mu}\right) .
$$

All other thermodynamic variables follow from Eq. (14). For example, the entropy (up to an irrelevant constant) is $S=\sum_{\alpha=1}^{N} \ln \left(\bar{n}_{\alpha}\right)$ which, in equilibrium, coincides with the expression derived in Ref. [19] by counting the number of ways in which a large number of "packets of power" can be distributed over the $N$ modes. The expression for $S$ served in Ref. [19] as the starting point for the development of optical thermodynamics. For instance, one can derive the following equation of state [19] $E-\mu A=N T$, which connects three extensive quantities $(E, A, N)$ to the two intensive variables $(\mu, T)$. It is important to keep in mind that in this analysis, the exact nonlinear form [e.g., Kerr in Eq. (1)] is completely irrelevant and its only role is to thermalize the system toward its equilibrium state.

\section{Fluctuations in the case of weak nonlinearities}

Next, we briefly discuss the fluctuations of the optical power $n_{\alpha}$ in the mode $\alpha$. If the nonlinearity, i.e., the intermode interaction, in the thermal equilibrium state is negligibly small $[19,26,27,29-31]$, then within the grandcanonical treatment the probability density for $n_{\alpha}$ is $\mathcal{P}\left(n_{\alpha}\right)=\left(1 / \mathcal{Z}_{\alpha}\right) \exp \left[-\beta\left(\varepsilon_{\alpha}-\mu\right) n_{\alpha}\right]$, where $\mathcal{Z}_{\alpha}$ is the normalization factor. This yields Eq. (13) for the average value $\bar{n}_{\alpha}$ and $\overline{\Delta n_{\alpha}^{2}}=\left(\bar{n}_{\alpha}\right)^{2}$ for the variance. The same results can be obtained, in an even simpler way, if one uses the expression $\Omega_{\alpha}=-T \ln \left(\pi T / \varepsilon_{\alpha}-\mu\right)$ for the contribution of mode $\alpha$ to the grand potential $\Omega$ [see Eq. (14)] and the standard formulas [64] $\bar{n}_{\alpha}=-\partial \Omega_{\alpha} / \partial \mu, \overline{\Delta n_{\alpha}^{2}}=T \partial \bar{n}_{\alpha} / \partial \mu$.

Thus, the standard deviation $\left(\overline{\Delta n_{\alpha}^{2}}\right)^{1 / 2} \equiv \sigma_{\alpha}$ comes out to be equal to the average optical power $\bar{n}_{\alpha}$. For a mode with macroscopic occupation $\left(n_{\alpha} \gg 1\right)$, this result looks paradoxical. The paradox, however, is well known in the theory of Bose-Einstein condensation and it is resolved by observing that we have here one of the rare cases when the canonical and the grand-canonical ensembles yield different results [64]. Indeed, in the experiment, as well as in our numerical simulations, the total optical power $\sum_{\alpha} n_{\alpha}=A$ is strictly conserved, while in the grand-canonical treatment it is conserved only on the average. This is perfectly fine for calculating various average quantities but not for the fluctuations. When the conservation law $\sum_{\alpha} n_{\alpha}=A$ is strictly enforced (canonical ensemble), the large unphysical fluctuations in a macroscopically occupied mode disappear (for instance, at $T=0$, when the entire power $A$ is located on a single mode, there are no fluctuations at all).

Note, however, that at high temperatures, when there are many modes populated with $\bar{n}_{\alpha} \lesssim 1$, the result $\sigma_{\alpha}=\bar{n}_{\alpha}$ does hold for such modes. This is because the constraint $\sum_{\alpha} n_{\alpha}=A \sim N$ on the total power does not significantly affect fluctuations in a single mode with $\bar{n}_{\alpha} \lesssim 1$ (the other modes serve as an "environment" for the mode $\alpha$ ). One should be aware of these large fluctuations when interpreting the numerical or the experimental data.

\section{MULTIMODE OPTICAL SYSTEMS WITH LONG-RANGE COUPLING}

\section{A. Connectivity matrix}

We consider the connectivity matrix $J_{l j}$ of the following form:

$$
J_{l j}=\left(\frac{J_{0}}{N}+\frac{\sigma}{\sqrt{N}} B_{l j}\right)\left(1-\delta_{l j}\right),
$$

where the first term describes a fully connected network, with equal couplings, while the second term introduces some randomness into the couplings. The origin of this long-range coupling is traced to index imperfections, or appropriately engineered cross-section deformations from an underlying ideal system (e.g., a cylindrical fiber [Fig. 1(d)] [25,51-53] or a circular disk resonator [Fig. 1(e)] [53-57]) when the equation of motion is written in the mode basis of the unperturbed system. Of course, in the basis of the modes of the perfect system, the nonlinear term in Eq. (1) will no longer have a local character. It rather acquires a form similar to the one appearing in the last term of the right-hand side of Eq. (2). Since, however, the exact form of the nonlinearity is irrelevant for the analysis of the thermal equilibrium state in the case of weak nonlinearities (see discussion at the end of Sec. II C), we have maintained its local nature for simplicity. Such simplification has allowed us to calculate analytically the magnetization of the photonic equilibrium state (see below). Moreover, it provided a confirmation for the validity of a mean-field theory of photonic networks that we present in Sec. IVA. 
It is an established method to analyze the connectivity matrix of Eq. (15) by separating it into a constant matrix, describing the mean coupling between the various modes, and a matrix representing the random fluctuations. Following a standard practice, we will model the random component of the couplings $B_{l j}=B_{j l}$ by a Gaussian distribution with zero mean and a unit standard deviation [65]. We point out that, unless stated otherwise, in all simulations below the random matrix elements $B_{i, j}$ remain the same (fixed) for a specific set of parameters $N, J_{0}, \chi$ dictating the Hamiltonian of the photonic network. For $\mathcal{H}$ in Eq. (4) to remain extensive, in the large $N$ limit, one has to scale the coupling strengths with $N$, as written in Eq. (15).

We can further analyze the spectral properties of the connectivity matrix Eq. (15). In the $\sigma=0$ limit it can be easily diagonalized. In this case the Hamiltonian Eq. (4) (with $\chi=0$ ) has one nondegenerate eigenvector (supermode) with eigenfrequency $\varepsilon_{1}=-(N-1) J_{0} / N$ and $(N-1)$-fold degenerate eigenvectors with frequency $\varepsilon_{\alpha}=J_{0} / N(\alpha=2, \ldots, N)$. In the opposite limit of $J_{0}=0$, the coupling matrix $J$ belongs to the "standard" Gaussian orthogonal ensemble of the random matrix theory (note though that it has zero diagonal elements). In this case its eigenvalues are distributed following the socalled Wigner semicircle probability distribution, $\rho(\epsilon)=$ $\left(1 / 2 \pi \sigma^{2}\right) \sqrt{4 \sigma^{2}-\epsilon^{2}}$, while the corresponding eigenvectors have random components following the so-called PorterThomas distribution [65]. The general case where both $J_{0}$ and $\sigma$ are different from zero interpolates between these two limits. For any arbitrary small $\sigma \ll J_{0}$, the $N-1$ degeneracy of the $\epsilon_{\alpha}=J_{0} / N$ levels of the constant coupling matrix will be removed and the corresponding eigenvectors will be those of an $(N-1) \times(N-1)$ Gaussian orthogonal ensemble random matrix. The $(N-1)$ time degenerate level is broadened into a band of half-width $2 \sigma$ and these levels follow a Wigner semicircle law. This picture is applicable as long as $2 \sigma<J_{0}$. It turns out that even for larger $\sigma$ the random matrix theory Wigner semicircle will still apply since, in this case, we can neglect the constant coupling matrix in Eq. (15). In the latter case, however, all levels are equally mixed and exchange energy without having any "outlier" isolated from the rest. This will become clear later on when we discuss the spin glass to ferromagnetic phase transition occurring in such cases (see Sec. III E).

The coupling matrix Eq. (15) gives rise to two distinct terms in the Hamiltonian Eq. (4). The first term resembles the Curie-Weiss model [66], where $N$ Ising spins are coupled to each other by constant distance-independent interactions. The second term resembles the SherringtonKirkpatrick model for a spin glass $[67,68]$, where the couplings are completely random. We write "resembles" because our model differs from the well-studied Curie-Weiss and Sherrington-Kirkpatrick models in three important respects: First, our dynamical variables $\psi_{l}$ are complex, as appropriate for the complex amplitudes of electric field, and they can be treated as real two-component "spins." Second, since $\left|\psi_{l}\right|^{2}$ is not restricted to some fixed value, our spins fluctuate not only in their direction but also in their size (note, though, that the nonlinearity does not allow for too wild fluctuations in size). And, third, the dynamics of our "photonic spins" conserve not only the energy (as in standard spin systems) but also the optical power; see Eq. (5). This second conservation law introduces novel features into the characteristics of the thermal equilibrium state: for instance, a condensation of optical power in a single mode.

Below we distinguish between the two limiting cases corresponding to a photonic network with equal couplings $(\sigma=0)$ and to its "random" coupling analog $\left(J_{0}=0\right)$. Then, we discuss the case where the connectivity matrix Eq. (15) contains both terms. We show that our photonic network exhibits various phases depending on the disorder strength of the coupling constants, the energy and optical power $(h, a)$ of the initial preparation, and the value of the nonlinearity parameter $\chi$.

\section{B. Numerical method}

The thermalization process of an initial state $\left\{\psi_{n}\right\}$ $(n=1, \ldots, N)$ has been investigated numerically using a high-order three-part split symplectic integrator scheme [69-71] for the integration of Eq. (1). The method conserved, up to errors $\mathcal{O}\left(10^{-8}\right)$, the total energy Eq. (4) and the optical power Eq. (5) of the system. These quantities have been monitored during the simulations in order to ensure the accuracy of our results.

We have focused our interest on the electric field amplitudes $\psi_{n}(t)$ and the supermode amplitudes $C_{\alpha}(t)$ which can be evaluated from the projection of $\psi_{n}(t)$ on the supermode basis; see Eq. (2). Knowledge of $C_{\alpha}(t)$ [or $\left.\psi_{n}(t)\right]$ allows us to calculate various thermodynamic quantities $\langle Q\rangle$ by making a time average and invoking ergodicity.

In practice, the approach to a thermal equilibrium state involves a long-time propagation of an initial preparation $\left\{\psi_{n}\right\}(n=1, \ldots, N)$. Typical integration times were as long as $4 \times 10^{8}$ inverse coupling constants. After an initial transient time $t_{\min }$, we have calculated a time-averaged value of the thermodynamic quantity of interest $Q$, i.e.,

$$
\langle Q\rangle_{t}=\frac{1}{t-t_{\min }} \int_{t_{\min }}^{t} Q(t) d t .
$$

A convergence of the observable $Q$ to a steady-state value has been considered as an (indirect) indication that the system has reached a thermal equilibrium state. An example of such simulations for a special case of a long-range coupling model Eq. (15) (see below for further analysis) consisting of $N=8$ nodes and constant couplings 


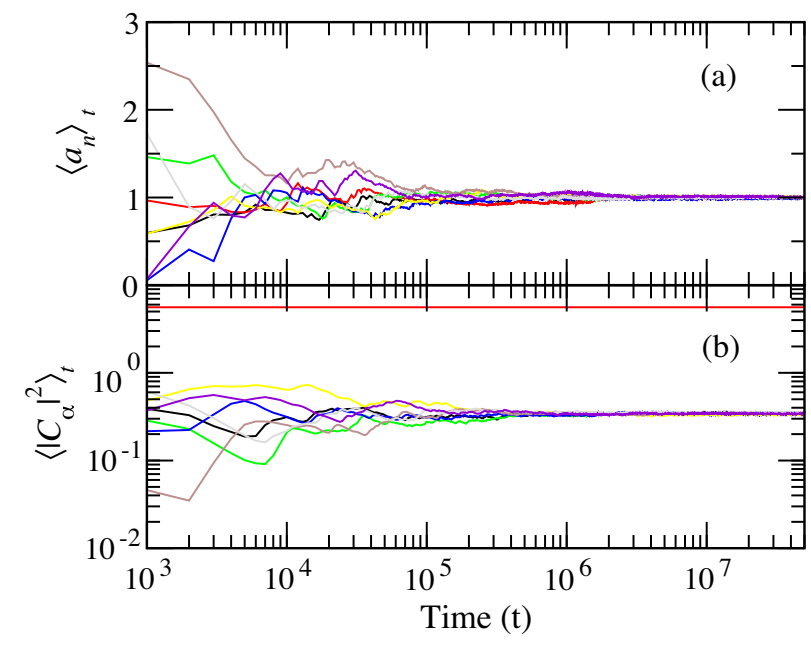

FIG. 2. An example (a) of a time-averaged nodal power $\left\langle a_{n}\right\rangle_{t}=$ $\left(1 /\left(t-t_{\min }\right)\right) \int_{t_{\min }}^{t}\left|\psi_{n}(t)\right|^{2} d t$ and (b) of a supermode power $\left\langle\left|C_{\alpha}\right|^{2}\right\rangle_{t}=\left(1 /\left(t-t_{\min }\right)\right) \int_{t_{\min }}^{t}\left|C_{\alpha}(t)\right|^{2} d t$. In these simulations the photonic network consists of $N=8$ nodes, $J_{0}=1.2$ and $\sigma=0$. Notice that the $\alpha=1$ mode (red line) is separated from the rest, saturating at a value of $\left\langle\left|C_{\alpha}\right|^{2}\right\rangle_{t} \approx 5$.6. The initial preparation has averaged energy density $h \approx-0.61$ and optical power $a=1$. The nonlinearity parameter is $\chi=0.01$. The initial time is $t_{\min }=$ 1000 in both cases.

$J_{0}=1.2(\sigma=0)$ are shown in Fig. 2. Specifically, in Fig. 2(a) we have taken $Q$ to be the nodal powers $a_{n}(t) \equiv\left|\psi_{n}(t)\right|^{2}$, while in Fig. 2(b) we considered $Q$ to be the supermode powers $\left|C_{\alpha}\right|^{2}$. In both cases, a clear convergence to a corresponding steady-state value is observed, indicating that the system has reached its equilibrium state.

In the case of weak nonlinearity the numerical results for $\left\langle\left|C_{\alpha}\right|^{2}\right\rangle$ have been compared against the theoretical predictions Eq. (13). A good agreement between them serves as a confirmation that the system reached a thermal equilibrium state. A disagreement between the numerics and the theoretical predictions of Eq. (13) indicates that the thermal equilibrium has not been reached. Instead, the system might have reached a metastable state as happens in the case of a spin-glass behavior [72,73]. Given enough time (large relaxation times), of course, the system will reach the global free-energy minimum.

In all cases, the initial conditions were generated by considering the field amplitudes $\left\{\left|\psi_{n}\right|\right\}$, and the phases $\phi_{n}$ being random numbers in the intervals $[1-\delta, 1+\delta]$ and $[-\pi, \pi]$, respectively. Out of a large number of $\left\{\psi_{n}\right\}$ configurations we have chosen only the ones that satisfy the energy and normalization constraints that define the specific state; see Eqs. (4) and (5), respectively. Finally, in all our simulations below we have used the normalization condition $A=\sum_{n}\left|\psi_{n}\right|^{2}=N$ [i.e., $a=1$; see Eq. (7)] associated with the total power.

\section{Equal-coupling networks}

We start our analysis with the equal-coupling photonic network. In this case each node is coupled to all other nodes by a hopping amplitude of equal strength. The Hamiltonian that describes this system is Eq. (4) with a connectivity matrix given by Eq. (15) with $\sigma=0$. We have

$$
\mathcal{H}\left\{\psi_{l}\right\}=-\frac{J_{0}}{N} \sum_{l \neq j} \psi_{l}^{*} \psi_{j}+\frac{1}{2} \chi \sum_{l}\left|\psi_{l}\right|^{4},
$$

where $l$ and $j$ run over all $N$ sites with the only constraint that $l \neq j$.

In the absence of nonlinearity the statistical mechanics of the system in Eq. (17) is trivial. Specifically, in the large- $N$ limit, only the lowest nondegenerate mode contributes to the total energy $E$ and, since the latter quantity is required to be extensive, it is clear that a final fraction of the total optical power $A$ must reside in that mode. A simple calculation, based on relations Eq. (11) and the expression Eq. (13), yields the following result: Since the total optical power is $A=a N$ and the total energy is $E=h N$, then, in the large- $N$ limit, the resulting chemical potential and the temperature are

$$
\mu=-J_{0}, \quad T=J_{0} a+h .
$$

Since, obviously, $J_{0} a \geq|h|$ must hold, the linear model does not allow for either negative temperatures or for a transition: a finite fraction $\left(|h| / J_{0} a\right)$ of the total power $A$ is condensed into the lowest mode [74].

A refined analysis, where the finite size effects are taken into consideration, leads to the following exact expressions for the chemical potential,

$$
\mu=-J_{0}\left[1-\left(a \frac{J_{0}}{h}+2\right) \frac{1}{N}+\left(a \frac{J_{0}}{h}\right) \frac{1}{N^{2}}\right],
$$

and the temperature,

$T=J_{0}\left[\left(a+\frac{h}{J_{0}}\right)-2\left(\frac{a}{N}\right)-\left(\frac{N-1}{N}\right) \frac{J_{0}}{h}\left(\frac{a}{N}\right)^{2}\right]$,

which, in the limit of $N \gg 1$, nicely match the results in Eq. (18). In Fig. 3 we have compared these theoretical predictions with the values of $(\mu, T)$ that we have extracted from our numerical simulations with the Hamiltonian of Eq. (17), using two multimode photonic networks with $N=8$ and 64 modes. The nice agreement indicates that these systems have reached a thermal equilibrium state. In the inset of Fig. 3 we also report $\left\langle\left|C_{\alpha}\right|^{2}\right\rangle_{t}$ [see Eq. (16)] by making use of the scaled variables $\hat{n}_{\alpha}$,

$$
\begin{aligned}
& \hat{n}_{1} \equiv\left(\left\langle\left|C_{1}\right|^{2}\right\rangle_{t}-a\right) / N=-h / J_{0}, \\
& \hat{n}_{\alpha} \equiv\left(\left\langle\left|C_{\alpha}\right|^{2}\right\rangle_{t}-a\right)(N-1) / N=h / J_{0},
\end{aligned}
$$




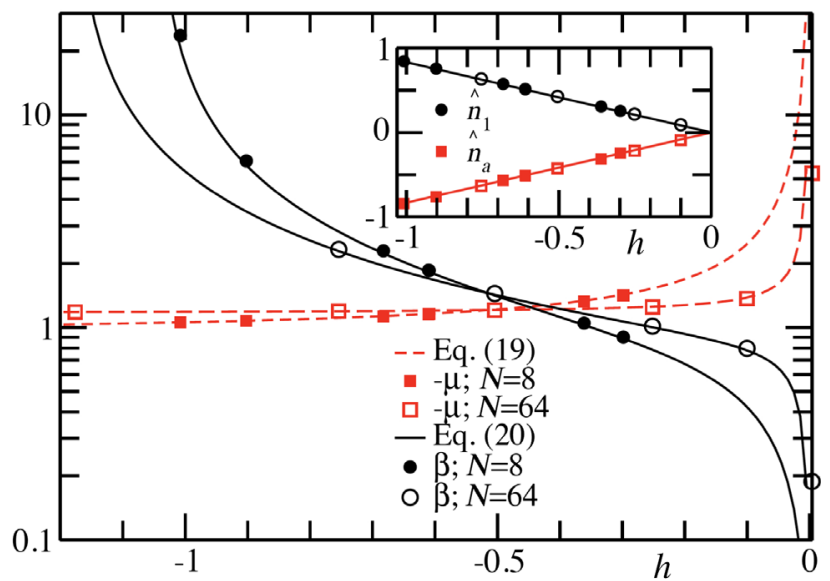

FIG. 3. The numerical values of chemical potential $-\mu$ (red squares) and inverse temperature $\beta=1 / T$ (black circles) versus the theoretical predictions Eq. (19) (red dashed line) and Eq. (20) (black lines), respectively. Filled symbols correspond to $N=8$, while open symbols correspond to $N=64$. Inset: The numerically extracted optical powers (symbols) scaled as $\hat{n}_{1}$ and $\hat{n}_{\alpha}$ versus the averaged energy density $h$. The rescaled $\hat{n}_{1}$ reaches the value zero at $h=h_{\max }$. The solid lines are the theoretical predictions of Eq. (13) while the two types of symbols correspond to $N=8$ (filled symbols) and $N=64$ (open symbols). In all cases we considered an initial optical power $a=1$, coupling constant $J_{0}=1.2$, while the nonlinearity is weak, $\chi=0.01$.

where in the second equation above $\alpha=2, \ldots, N$. The right-hand side of Eqs. (21) has been evaluated using Eq. (13) together with Eqs. (11). It is important to point out that thermalization has been achieved even for the system with relatively small number of nodes, $N=8$.

The presence of nonlinearity changes the picture completely and provides us with an example of a (mean-field) optical phase transition from an ordered to a disordered phase. In the former phase the amplitudes $\psi_{l}$ on different nodes are correlated, while in the latter phase the nodes become essentially decoupled from each other. The ordered (disordered) phase corresponds to low (high) temperature, i.e., to small (large) internal energy $E$.

The ground state of the Hamiltonian (17) corresponds to a uniform field configuration $\left\{\psi_{l}\right\}=\sqrt{A / N}(1,1, \ldots, 1)$, where the normalization is such that the total optical power is $\sum_{l}\left|\psi_{l}\right|^{2}=A$. All the "spins" in this state point in the same direction. Note that the ground state is highly degenerate: one can rotate all the spins by an angle $\theta$, i.e., multiply the field by an overall phase factor $\exp (i \theta)$. The ground-state energy is

$$
E_{\min }=-J_{0} A+\frac{1}{2} \chi A^{2} / N=\left(-J_{0} a+\frac{1}{2} \chi a^{2}\right) N,
$$

corresponding to $T=0$. In the opposite limit, $T \rightarrow \infty$, the kinetic energy (hopping) can be neglected and the system reduces to a set of uncoupled nonlinear oscillators. The probability density to find a node with optical power $|\psi|^{2} \equiv$ $I$ is $p(I) \sim \exp \left(\beta \mu I-\frac{1}{2} \beta \chi I^{2}\right)$, and a simple calculation yields an average thermal energy equal to $\chi a^{2}$, in the $T \rightarrow \infty$ limit. Thus, the maximal energy of the system [see Eq. (10)] is $E_{\max }=\chi a^{2} N$, indicating that for fixed $a$ the thermalization occurs for $E_{\min }<E<E_{\max }$. One can, of course, endow the system with an energy larger than $E_{\max }$, for instance, by putting all the norm $A$ on a single site (in which case the energy $E$ would become "superextensive," proportional to $N^{2}$ ). We do not consider, however, initial preparation with $E>E_{\max }$ since we do not address the "non-Gibbsian" region in the $(E, A)$ plane where breathers can be formed due to strong nonlinearities (see previous discussion and also Refs. [58,59]).

Next, we proceed with the calculation of the partition function Eq. (6) which is performed using the saddle-point method. It is convenient to write the complex amplitudes $\psi_{l}=q_{l}+i p_{l}$, where $\left(q_{l}, p_{l}\right)$ is a pair of real variables. The Hamiltonian

$\mathcal{H}\left\{q_{l}, p_{l}\right\}=-\frac{J_{0}}{N} \sum_{l, j}\left(q_{l} q_{j}+p_{l} p_{j}\right)+\frac{\chi}{2} \sum_{l}\left(q_{l}^{2}+p_{l}^{2}\right)^{2}$

can be interpreted in terms of interacting two-component spins. We write $\sum_{l, j} q_{l} q_{j}=\left(\sum_{l} q_{l}\right)^{2}$ and use the identity

$$
\begin{aligned}
& \exp \left[\frac{\beta J_{0}}{N}\left(\sum_{l} q_{l}\right)^{2}\right] \\
& =\sqrt{\frac{N \beta J_{0}}{\pi}} \int_{-\infty}^{\infty} d x \exp \left[\beta J_{0}\left(-N x^{2}+2 \chi \sum_{l} q_{l}\right)\right],
\end{aligned}
$$

and similarly for $\sum_{l, j} p_{l} p_{j}$. The integrals over $q_{l}, p_{l}$ in the partition function now factorize into a product of integrals, each involving only variables for one node. Furthermore, in the large- $N$ limit, the grand-canonical partition function $\mathcal{Z}$ is dominated by a saddle point, which can be interpreted as the order parameter, i.e., the average field $\bar{\psi}$ (the magnetization in the statistical mechanics language). Actually, there is a whole family of saddle points, distinguished from each other by an overall phase. Choosing one saddle point of the family amounts to breaking the rotational symmetry in the spin space, obtaining a nonzero value for the order parameter. We chose $\bar{\psi} \equiv m$ to be real. Skipping all calculation details, we only give the final equation for $m$ :

$$
m=\frac{1}{2 \beta J_{0} F(m)} \frac{d F(m)}{d m} \equiv Q(m),
$$

where the function $F(m)$ is given by the integral $F(m)=$ $2 \pi \int_{0}^{\infty} r d r I_{0}\left(2 \beta J_{0} m r\right) \exp \left(\beta \mu r^{2}-\frac{1}{2} \chi \beta r^{4}\right)$ and $I_{0}(x)$ is the modified Bessel function of order zero. 
For small $m, Q(m)$ is a linear function of $m$, $Q(m)=s m$, and the slope $s$ determines whether Eq. (25) has a nontrivial solution $m \neq 0$. Such a solution exists only if $s>1$. The slope can be calculated using the small argument expansion $I_{0}(x)=1+\frac{1}{4} x^{2}$, and it can be written as $s=\beta J_{0} K_{3} / K_{1}$, where an integral $K_{n}$ is defined as $K_{n}=\int_{0}^{\infty} d r r^{n} \exp \left(\beta \mu r^{2}-\frac{1}{2} \chi \beta r^{4}\right)$.

Let us, as an example, fix $\mu$ at the value zero and study $s$ as a function of the temperature $T=1 / \beta$. For $\mu=0$ a simple expression for the slope $s$ is obtained; namely, $s=J_{0}(2 / \pi T \chi)^{1 / 2}$. The value $s=1$ yields the critical temperature $T_{c}=2 J_{0}^{2} / \pi \chi$. For temperature $T$ slightly below $T_{c}$ one finds, by keeping the term of order $m^{3}$ in the expansion of $Q(m)$, the standard mean-field result $m \sim\left(T_{c}-T\right)^{1 / 2}$. When the temperature decreases further, toward $T=0$, the magnetization increases and approaches the maximal value, corresponding to the fully ordered ground state. Taking again $\mu=0$ as an example, one obtains from Eq. (25) that, in the $\beta \rightarrow \infty$ limit, $m \rightarrow \sqrt{J_{0} / \chi}$. This result becomes transparent when written in terms of the optical power $A$. Indeed, at $T=0$ the total power resides in the (fully correlated) ground state, so that the magnetization per site is $m=\sqrt{A / N} \equiv \sqrt{a}$. To connect $a$ to $\mu$ we have to use the expression Eq. (22) for the ground-state energy, which yields $\mu=(1 / N) \partial E / \partial a=$ $-J_{0}+\chi a$. For $\mu=0$, one obtains $m=\sqrt{a}=\sqrt{J_{0} / \chi}$.

Thus, Eq. (25) is well suited for studying $m$, as a function of $\beta$, for a fixed value of $\mu$. In experiment, however, one usually controls the optical power $A$, rather than the conjugate variable $\mu$. Calculating analytically $m$ as a function of $\beta$ for fixed $A$ is more involved than the above calculation for fixed $\mu$, and we do not attempt it in the present paper. Instead, in Fig. 4 we present some numerical results, serving a double purpose: first to verify that the system, with the appropriate initial preparation, indeed thermalizes, and then to study its properties in the thermal equilibrium state as a function of the averaged energy density $h$. To this end, we evaluate numerically the timeaveraged magnetization $\bar{m}$ [see Fig. 4(a)] defined as

$$
\bar{m}=\left\langle\left|\frac{1}{N} \sum_{n} \psi_{n}(t)\right|\right\rangle_{t}
$$

In our numerics, we consider moderate values of the nonlinear parameter $\chi=1$ and coupling constant $J_{0}=1.2$. At the ground state $h=h_{\min }$ all "spins" have the same orientation. As a result, the magnetization acquires its maximum value $\bar{m}=1$, indicating a ferromagnetic behavior. For higher $h$ values the magnetization decreases, and at $h \rightarrow h_{c} \approx 0.75$, which is smaller than $h_{\max }=1$, it acquires a constant value $\bar{m}=\mathcal{O}(1 / \sqrt{N})$ [see inset of Fig. 4(a)], associated with finite size effects. Further numerical analysis indicates that in the thermodynamic limit of $N \rightarrow \infty$ the magnetization, as a function of

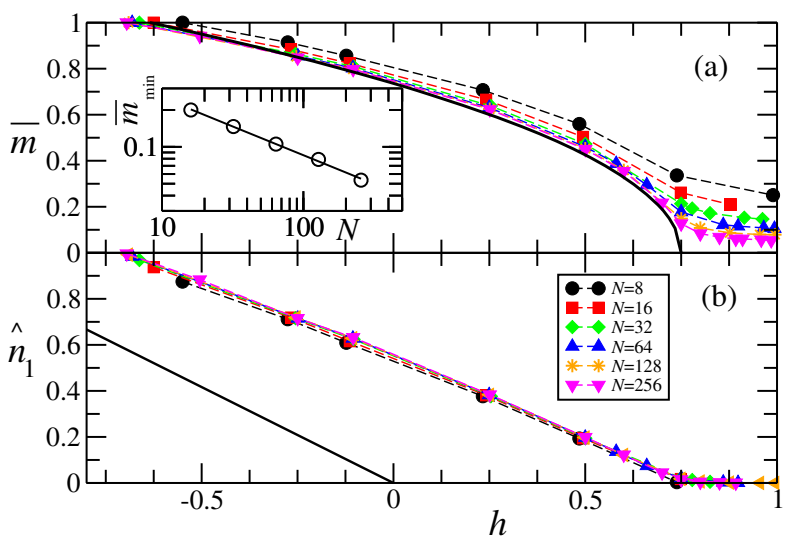

FIG. 4. (a) The time-averaged magnetization versus the averaged energy density $h$. We have simulated various multimode photonic networks described by Eq. (17) with $N=8,16,32,64$, 128,256 . The bold solid line is the best asymptotic $(N \rightarrow \infty)$ fit indicating a square-root singularity of the magnetization, i.e., $\bar{m} \approx 0.85 \sqrt{0.75-h}$. Inset: The asymptotic value $\bar{m}_{\text {min }}$ of timeaveraged magnetization (circles) versus the number of nodes $N$. The solid line is the best fit to the numerical data and demonstrates a convergence to zero as $\bar{m}_{\min } \approx 0.75 / \sqrt{N}$. (b) The time-averaged optical power of the ground-state supermode for networks of different number of nodes $N$. We have used the same scaling variable $\hat{n}_{1}$ as in Fig. 3. The condensation transition, corresponding to $\hat{n}_{1}=0$, occurs for the same value of $h=h_{c} \approx 0.75$ as the one that signifies the transition from a ferromagnetic to a paramagnetic behavior in (a). In all cases we have considered an initial averaged optical power $a=1$, coupling constant $J_{0}=1.2$, while the nonlinearity is $\chi=1$. In this case, the maximum energy density is $h_{\max }=1$.

$h$, approaches zero following a square-root behavior $\bar{m} \approx$ $0.85 \sqrt{h_{c}-h}$ [see bold black line in Fig. 4(a)]. Such behavior is characteristic of a second-order phase transition, from a "ferromagnetic" to a "paramagnetic" (optical) phase.

In Fig. 4(b) we show the numerical results for the timeaveraged optical power in the ground-state supermode $\left\langle\left|C_{1}\right|^{2}\right\rangle_{t}$ versus the averaged energy density of the initial beam. In the simulations, we have considered the same photonic networks as above with $\chi=1, J_{0}=1.2$, and different numbers of nodes $N$. The numerical findings are reported using the scaled variable $\hat{n}_{1}$; see Eq. (21). We find that for low averaged energy densities $h$, the optical power of the ground state is $\left\langle\left|C_{1}\right|^{2}\right\rangle_{t} \approx N$, indicating a condensate. As $h$ increases, the condensate depletes, and eventually at $h=h_{c}$, corresponding to the ferromagnetic-paramagnetic transition, it is completely destroyed, i.e., $\hat{n}_{1}=0$. In the same figure we also report for comparison the theoretical value of $\hat{n}_{1}=-h / J_{0}$ (black solid line), applicable for the case of weak nonlinearities; see Eq. (13). We stress once more that the condensation transition analyzed above is due solely to the nonlinearity, unlike the previous studied cases where the transition occurred already in the linear system [29,32]. 


\section{Random coupling}

Next, we analyze the effect of disorder in the coupling constants of the photonic network, i.e., $\sigma \neq 0$ in Eq. (15). First, we consider the case of extreme disorder where $J_{0}=0$. In this case the off-diagonal elements of $J$ have equal probabilities to be positive or negative; i.e., the system is completely "frustrated." Despite the vast literature on spin networks, this model with complex, "soft spins" has not been studied up to now. Of course, certain analogies with the Sherrington-Kirkpatrick model can still be instructive. In the latter case, there is a transition from a paramagnetic phase to a spin-glass phase when the temperature (the energy $E$ of the system) decreases. One characteristic distinction between the two phases is that for the spin glass the thermalization time is much longer than for the paramagnet. Moreover, for large $N$ a spin glass does not reach a full thermal equilibrium in any reasonable time, and the system gets stuck in one of the many metastable states.

Our simulations for the random coupling multimode photonic network are presented in Fig. 5. For a weak nonlinearity $\chi=0.01$, the equilibrium optical powers $\bar{n}_{\alpha}$ of the supermodes (of the linear problem) are given by Eq. (13). In the simulation we evaluated the set $\left\{\left|C_{\alpha}(t)\right|^{2}\right\}$ as a function of time, extracted their time average Eq. (16), and use their comparison to Eq. (13) as a criterion for thermalization. We find that for energy $h \approx-0.685$, see Fig. 5(a), the system eventually gets close

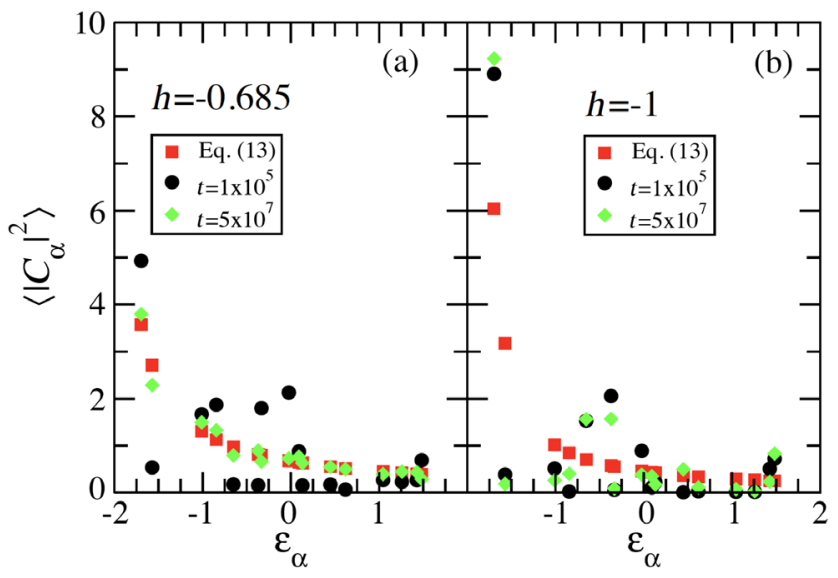

FIG. 5. Comparison between the time-averaged supermode optical powers for a multimode photonic network whose dynamics is described by the Hamiltonian Eq. (4) with random connectivity matrix Eq. (15) with $J_{0}=0$ and $\sigma=1$ and the theoretical results (red squares) of Eq. (13) for weak disorder. Black circles correspond to moderate time evolutions with $t=10^{5}$ while green diamonds correspond to larger time evolutions with $t=5 \times 10^{7}$. The initial state has (a) high averaged energy density per mode $h=-0.685$ (corresponding to high temperatures) or (b) low averaged energy density per mode $h=-1$ (corresponding to moderate or low temperatures). In both cases the average optical power is $a=1$. In these simulations, the number of modes is $N=16$ and the nonlinear parameter is $\chi=0.01$. to thermal equilibrium at times $t \approx 5 \times 10^{7}$ (in units of standard deviation of the coupling elements). After this time the occupation numbers change only slightly. For higher energies (not shown), the thermalization time becomes shorter (for example, for $h \approx-0.15$ the thermalization time for a network of $N=16$ nodes was $\left.\propto 10^{4}\right)$. On the other hand, for energy $h=-1$ [see Fig. 5(b)], the optical powers $\left\{\left\langle\left|C_{\alpha}\right|^{2}\right\rangle_{t}\right\}$ are far away from $\left\{\bar{n}_{\alpha}\right\}$ even after a fairly long time $t=5 \times 10^{7}$ and, moreover, they do not show any significant change with respect to the results extracted for shorter times $t=10^{5}$ [filled black circles in Fig. 5(b)]. We have confirmed that the lack of thermalization (for any reasonably large time) is typical for other initial preparations (with the same $h$ ). This is a typical behavior of a spin glass.

Indeed, the most important signature of a spin glass, from which the term itself was derived, is that at low temperatures the directions of spins at various sites get frozen in some random configuration (metastable state). For our "optical spin glass" such behavior implies that the average values of the complex amplitudes $\left\{\psi_{l}\right\}$, and in particular the phases $\left\{\theta_{l}\right\}$, form a random set. The "average" here refers to the thermal statistical average, for a fixed realization of the disorder. One could expect that in a numerical simulation averaging over a statistical ensemble can be replaced by the time average. However, due to a dynamic overall phase in the time evolution defined in Eq. (1), the time average of the phase $\theta_{l}$, at any site $l$, amounts to zero. Therefore, in order to distinguish a spin glass from a paramagnet, we use the following criterion. Let us denote by superscripts $\alpha, \beta$ two initial preparations, with the same total energy $E$ and optical power $A$. Their time evolution is given by $\left\{\psi_{l}^{\alpha}(t)\right\}$ and $\left\{\psi_{l}^{\beta}(t)\right\}$, respectively. The quantity $\zeta(t)=$ $(1 / N) \sum_{l}\left(\psi_{l}^{\alpha}(t)\right)^{*} \psi_{l}^{\beta}(t)$ is a measure of the overlap between the two evolutions, at time $t$. In the paramagnetic phase $\zeta(t)$ decreases with time, approaching zero, because (in the large- $N$ limit) the two evolutions become completely uncorrelated.

It is appropriate to consider many initial preparations, i.e., many $(\alpha, \beta)$ pairs, and treat the real and the imaginary parts of $\zeta(t)$ as statistical quantities with probability distribution $\mathcal{P}(\operatorname{Re}(\zeta))$ and $\mathcal{P}(\operatorname{Im}(\zeta))$. In the long-time limit, and for large but finite $N$, these distributions are expected to have a manifestly different form in the two phases: For a paramagnet, $\mathcal{P}(\operatorname{Re}(\zeta))$ and $\mathcal{P}(\operatorname{Im}(\zeta))$ should be narrow distributions (with a width approaching zero when $N \rightarrow \infty$ ), centered around $\zeta=0$. These expectations are confirmed by our numerical data, which are reported in Figs. 6(a) and 6(b) for photonic multimode networks with initial preparations having high values of averaged energy density $h=-0.155$. An increase of the number of modes $N$ leads to narrower distributions around $\zeta=0$. When, on the other hand, we consider the same distributions for a set of initial preparations with low value of $h=-1.4$, 

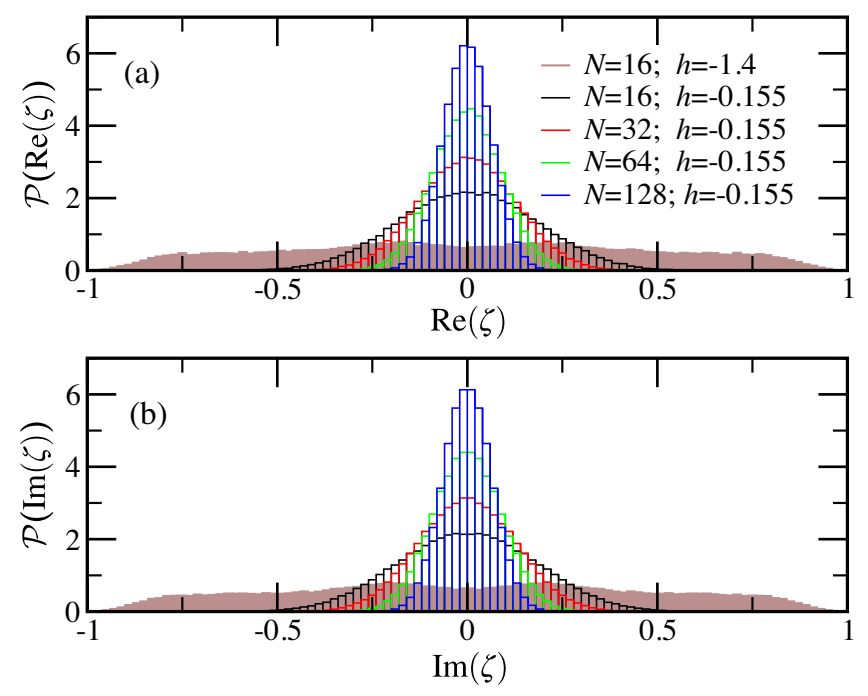

FIG. 6. Distribution of the (a) real and the (b) imaginary part of the correlation function $\zeta(t)$. The "empty" histograms correspond to initial preparations with averaged energy density, which is $h=$ -0.155 (high-energy regime). The brown "filled" histogram corresponds to a network with $N=16$ and initial preparations with averaged energy density, which is $h=-1.4$ (low-energy regime; the smallest eigenvalue of the linear system was approximately -1.7 ). The number of modes are indicated by the color of the histogram in the inset. The average optical power was kept constant $a=1$ while $\chi=0.01$. In all these simulations the integration time was as long as $t=10^{5}$ and we have generated more than $32 \times 10^{4}$ correlations $\zeta$ for the statistical processing.

we observed an entirely different behavior for $\mathcal{P}(\operatorname{Re}(\zeta))$ and $\mathcal{P}(\operatorname{Im}(\zeta))$ [see brown highlighted histogram in Figs. 6(a) and 6(b)]. Namely, they become broad and almost flat, covering the whole allowed interval, i.e., $-1<\operatorname{Re}(\zeta), \operatorname{Im}(\zeta)<1$. We stress that the above simulations were performed for a given realization of disorder and for the same energy, $h=-1.4$. Only the initial preparations have been randomly chosen. We interpret the "flatness" of $\mathcal{P}(\operatorname{Re}(\zeta)), \mathcal{P}(\operatorname{Im}(\zeta))$ as a signature of many metastable states, typical of a spin glass [72,73].

\section{E. Mixed coupling schemes}

It is natural to ask what happens to the network at low averaged energy densities $h$ when both terms in the connectivity matrix Eq. (15) coexist, i.e., $J_{0} \neq 0$ and $\sigma \neq 0$. In Fig. 7 we report the dependence of the magnetization $\langle|m|\rangle_{t}$ versus the control parameter $x=J_{0} / \sigma$ and for $h \approx 0.88 \epsilon_{1}\left(\epsilon_{1}\right.$ is the smallest eigenvalue of the corresponding linear Hamiltonian). In the simulations we keep $\sigma=1$ and change the magnitude of $J_{0}$. Following the same scheme as in Sec. III C, we break the rotational symmetry of the spin space by preparing the system at a real-valued configuration $\left\{\psi_{n}\right\}$. When $x=0$, the connectivity matrix has only random coupling elements (i.e., $J_{0}=0$ ) "forcing" the network into the spin-glass phase. In this regime, the

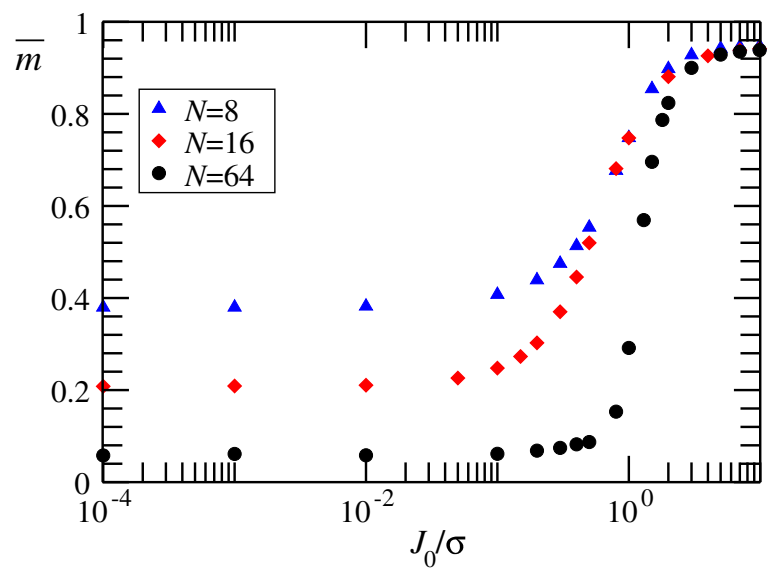

FIG. 7. The time-averaged magnetization versus the control parameter $x=J_{0} / \sigma$. The transition at $x \approx 1$ becomes more abrupt as $N$ increases. In these simulations, the initial preparation was taken to have averaged energy density $h \approx 0.88 \epsilon_{1}$ (low-energy regime; $\epsilon_{1}$ is the smallest eigenvalue of the corresponding linear system) while the nonlinearity is $\chi=0.01$.

system evolves toward a metastable state with the "spins" pointing toward random directions. As a result, the magnetization is approaching zero as $\bar{m} \propto \mathcal{O}(1 / N)$ in the limit of large- $N$ values; see Fig. 7. In the other limiting case of $x \rightarrow \infty$ the randomness in the coupling elements is suppressed and the connectivity matrix is dominated by (essentially) constant couplings $J_{0}$. In this case, the network is in the ferromagnetic phase and the magnetization acquires a nonzero magnitude $\bar{m} \neq 0$ which is dictated by the value of $h$ (e.g., for $h=h_{\min } \approx-J_{0}$ the magnetization is $\bar{m}=1$ ). Our analysis (see Fig. 7) indicates that the transition from a spin-glass to a ferromagnetic phase occurs at $x \sim 1$. The transition becomes more abrupt, as expected from statistical mechanics, in the limit of large- $N$ values.

\section{MULTIMODE OPTICAL SYSTEMS WITH NEAREST-NEIGHBOR COUPLING}

Many photonic networks, like the ones shown in Figs. 1(a)-1(c) are described by Eq. (4), where the index $j$ in the first sum on the right-hand side enumerates nearest neighbors. In this section we address the phase transition or crossover between different phases in such type of systems, utilizing a mean-field theory approach and a scaling theory which will allow us to establish a universality for the magnetization of photonic networks. Bellow, we assume that the coupling coefficients are constants; i.e., $J_{i, j}=J_{0}$ for nearest neighbors and zero otherwise.

\section{A. Mean-field theory of photonic networks}

To be specific, let us consider a lattice of coupled resonators with coordination number $n_{c}$; see Fig. 1(b). For the mean-field analysis, we introduce the average field value $\bar{\psi}$ (the order parameter) and the field fluctuations 
$\delta \psi_{l}=\psi_{l}-\bar{\psi}$ at each resonator $l$. Writing the complex amplitudes as $\psi_{l}=\bar{\psi}+\delta \psi_{l}$ and substituting in Eq. (4) while neglecting terms of order $\mathcal{O}\left(\delta \psi^{2}\right)$, we get the meanfield Hamiltonian $\mathcal{H}_{\mathrm{MF}}$ :

$$
\begin{aligned}
\mathcal{H}_{\mathrm{MF}}\left\{\psi_{l}(t)\right\}= & -n_{c} J_{0}\left(\bar{\psi} \sum_{l}^{N} \psi_{l}^{*}+\bar{\psi}^{*} \sum_{j}^{N} \psi_{j}-N|\bar{\psi}|^{2}\right) \\
& +\frac{1}{2} \chi \sum_{l}^{N}\left|\psi_{l}\right|^{4}
\end{aligned}
$$

where $N$ is the total number of resonators. Equation (27) is a sum of $N$ independent Hamiltonians whose grand partition function takes the form

$$
\mathcal{Z}=I^{N}(\bar{\psi}) \exp \left(-n_{c} \beta J_{0} N|\bar{\psi}|^{2}\right)
$$

where

$$
\begin{aligned}
I(\bar{\psi})= & \int d \psi d \psi^{*} \exp \left(\beta \mu|\psi|^{2}+n_{c} \beta J_{0}\left(\bar{\psi} \psi^{*}\right.\right. \\
& \left.\left.+\bar{\psi}^{*} \psi\right)-\frac{1}{2} \beta \chi|\psi|^{4}\right) .
\end{aligned}
$$

The order parameter $\bar{\psi} \equiv m$ is identified with the magnetization (we break the symmetry by taking $\bar{\psi}$ to be real and positive, although in general it is a complex function, i.e., $\left.\bar{\psi}=|\bar{\psi}| e^{i \bar{\theta}}\right)$. Then, writing $\psi=r e^{i \theta}$ and moving to the $(r, \theta)$ variables, Eq. (29), after integration over $\theta$, yields

$$
I(m)=2 \pi \int_{0}^{\infty} r I_{0}\left(2 n_{c} \beta J_{0} m r\right) \exp \left(\beta \mu r^{2}-\frac{1}{2} \beta \chi r^{4}\right) d r,
$$

which, after substitution back into Eq. (28), allows us to evaluate the grand partition function and the grand potential $\Omega(\beta, \mu, m)=-(1 / \beta) \ln \mathcal{Z}$. The latter takes the form

$$
\Omega(\beta, \mu, m)=\frac{N}{\beta}\left[\beta J_{0} m^{2}-\ln I(m)\right] \equiv \frac{N}{\beta} g(m) .
$$

Finally, the equation for the magnetization $m$ is obtained by minimizing $\Omega$, i.e., the function $g(m)$ :

$$
\frac{d g}{d m}=2 \beta J_{0} m-\frac{1}{I(m)} \frac{d I(m)}{d m}=0,
$$

which is essentially the same equation as Eq. (25). The only difference is that the coordination number of the network $n_{c}$ appears in some coefficients in front of the integration variable $\psi$ in Eq. (29). This discrepancy leads to a critical temperature that depends on the coordination number $n_{c}$ without, however, altering the physics or the critical exponents associated with the phase transitions.

Of course, the mean-field theory is not applicable for low-dimensional systems (e.g., in one-dimensional systems there can be no thermodynamic transition at all). In many cases, however, mean-field theory is a good starting point for the study of phase transitions, and sometimes it gives accurate quantitative results-the most famous example being the Ginsburg-Landau theory of superconductivity (incidentally, the order parameter there is a complex function as in our case). For example, one can check that in the case of long-range coupling (corresponding to $n_{c} \rightarrow N$ and $J_{0} \rightarrow J_{0} / N$ ), the expression Eq. (32) reproduces exactly the results of the previous section.

\section{B. Universal scaling theory for one-dimensional photonic networks}

Next we consider a one-dimensional nearest-neighbor coupling photonic network [e.g., Fig. 1(a)] with coordination number $n_{c}=2$. Our analysis focuses on the magnetization $\bar{m}$ and its behavior with respect to the parameters $\left(h, a, \chi, J_{0}, N\right)$ that control the state of the photonic network. To this end, we have developed a one-parameter scaling theory for $\bar{m}$ and show the existence of a universal scaling function that controls the variation of magnetization as the various parameters $\left(h, a, \chi, J_{0}, N\right)$ vary. Specifically, we show that

$$
\frac{\bar{m}}{\sqrt{a}}=f(\Lambda \equiv N / \xi) \approx \begin{cases}\mathcal{O}(1) & \text { for } \Lambda \ll \mathcal{O}(1) \\ \Lambda^{-1 / 2} & \text { for } \Lambda \gg 1,\end{cases}
$$

where $\xi$ is the so-called correlation length and $f(\Lambda)$ is a universal function, that depends only on $\Lambda$. The scaling relation Eq. (33) describes nicely the crossover from a "quasiferromagnetic" state of the network to a paramagnetic state.

The important quantity for establishing Eq. (33) is the correlation length, which encodes all information associated with the various parameters that defined the photonic network, i.e., $\xi=\xi\left(h, a, \chi, J_{0}\right)$. It describes the characteristic length scale over which the photonic spins are strongly correlated, roughly, pointing in the same direction. Formally, it is defined via the decay rate of the spin-spin correlation function $\mathcal{C}_{r}$ :

$\mathcal{C}_{r} \equiv \frac{1}{2 N a}\left\langle\sum_{n}\left(\psi_{n}^{*} \psi_{n+r}+\text { c.c. }\right)\right\rangle_{t} \sim \exp (-r / \xi)$.

The correlation length is finite (unless the temperature $T$ is strictly zero), but it is macroscopic (many lattice spacings) if $T$ is sufficiently low. Thus, although there is no phase transition in a strict sense, one can observe a crossover between a quasiferromagnetic and paramagnetic states [75].

Let us first consider the scenario where $N \ll \xi$. In this case, the network consists of a single coherent unit of $N$ 


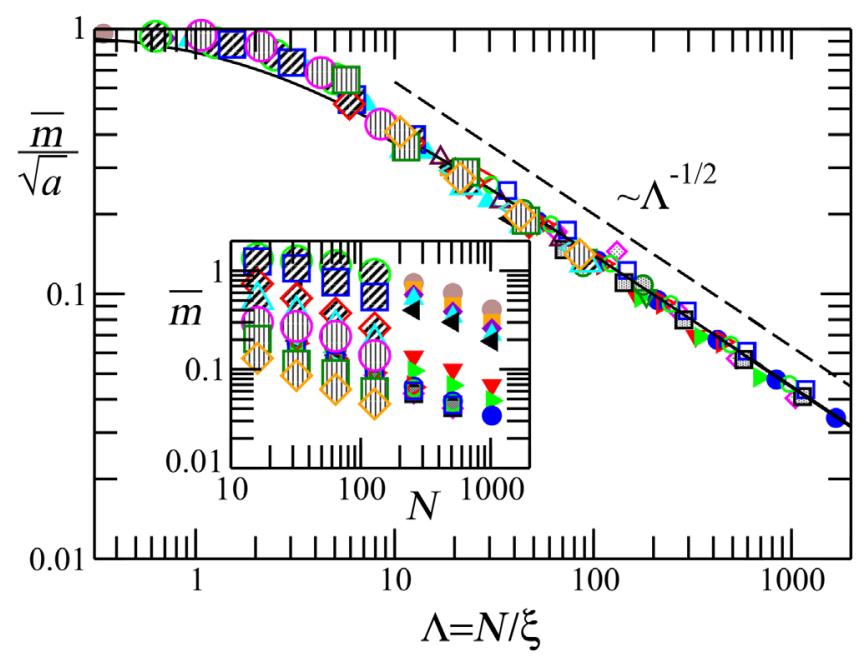

FIG. 8. Scaled time-average magnetization $\bar{m} / \sqrt{a}$ versus the scaling parameter $\Lambda=N / \xi$ for a one-dimensional photonic network with constant $\left(J_{0}=1\right)$ nearest-neighbor couplings (coordination number $n_{c}=2$ ). The various symbols and colors indicate different set of nonlinearities $\chi$ (full symbols, $\chi=1$, $a=1$; open symbols, $\chi=0.1, a=1$; partially filled symbols, $\chi=0.01, a=1$; diagonally striped filled enlarged symbols, $\chi=1, a=2$; vertically striped filled symbols, $\chi=1, a=0.1$ ) and various $h$ values. The black solid line indicates Eq. (35). The dashed black line has slope $\Lambda^{-1 / 2}$ and it is drawn to guide the eye. In the inset we report the unscaled data for $\bar{m}$.

spins, pointing in the same direction (the direction, of course, is changing with time). Since the magnitude of each spin is of order $\sqrt{a}$, the magnitude of the total magnetic moment $M_{N}$ of the sample is proportional to $N \sqrt{a}$, and thus, the typical value of the magnitude of the magnetization is $\bar{m} \sim \sqrt{a}$.

On the other hand, when $N \gg \xi$, the photonic network consists of $N / \xi=p$ uncorrelated domains. The magnetic moment of the $\alpha$ th domain $(\alpha=1, \ldots, p)$ is represented by a two-dimensional vector $\vec{M}_{\alpha}$ with a length of order $\xi \sqrt{a}$ and random direction. Estimating the magnetic moment $\vec{M}_{N}$ of the entire network amounts to summing up $p$ random two-dimensional vectors (phasors). Neglecting small fluctuations in the length of different $\vec{M}_{\alpha}$, and assuming completely random directions (phases), one obtains a typical value for the magnetization of the total network, which is $\bar{m} \sim M_{N} / N=(\xi \sqrt{a}) \sqrt{p} / N=$ $\sqrt{a}(N / \xi)^{-1 / 2}$.

We are now in a position to formulate a scaling theory that dictates the "evolution" of the rescaled magnetization $(\bar{m} / \sqrt{a})$ of the one-dimensional photonic network with coordination number $n_{c}=2$. Specifically, combining the results of the previous analysis for the two limiting cases, we postulate Eq. (33), which indicates that the rescaled magnetization $\bar{m} / \sqrt{a}$ is described by a universal, oneparameter scaling function where the temperature, as well as other properties of the system $\left(h, a, \chi, J_{0}, N\right)$, enter into $\bar{m} / \sqrt{a}$ only via the ratio $\Lambda=N / \xi$. This is a manifestation of universality: the behavior of a macroscopic scale is insensitive to the microscopic details characterizing the system.

Our numerical results reported in Fig. 8 support nicely the scaling ansatz Eq. (33). A possible interpolating law valid in all regimes (including the crossover region) is

$$
f(\Lambda)=\sqrt{\frac{a_{0}}{\Lambda+a_{0}}},
$$

where comparison with the numerical data in the two limits yields the fitting parameter $a_{0} \approx 2$. The resulting analytical formula (see solid line in Fig. 8) nicely fits the numerical data in all regions, and therefore provides a compact summary of the crossover of the magnetization from the ferromagnetic to the paramagnetic phase.

\section{CONCLUSIONS}

We unveiled a connection between nonlinear photonic networks, consisting of many coupled single modes, and spin networks. As opposed to standard spin models, our "photonic spins" are complex, soft (i.e., their size fluctuates), and their dynamics has two constants of motion: the total energy and the total optical power. This second conservation law is responsible for the appearance of novel optical phase transitions and the emergence of new forms of thermal photonic states. We found that these transitions are controlled by the nature of the connectivity of the network (long range, nearest neighbors, disorder, or constant couplings) and the amount of the averaged energy density of the initial optical preparation. Another important parameter is the nonlinearity that controls the thermalization process.

For one-dimensional networks with constant nearestneighbor couplings we established a universal oneparameter scaling theory that describes their crossover from "quasiferromagnetic" to a paramagnetic phase. In contrast, using a mean-field approach, we show that photonic lattices with constant nearest-neighbor couplings demonstrate in the nonlinear regime a phase transition from a ferromagnetic to a paramagnetic phase as the averaged energy density $h$ of an initial preparation increases. This transition is also present in the case of long-range photonic networks with constant couplings and it is associated with the destruction of a photonic condensate and its depletion to thermal states. In the other limiting case of random coupling constants a long-range coupling photonic network is driven from a paramagnetic to a spin-glass phase as $h$ decreases. Finally, we show that the same long-range coupling network, when prepared at low energies, undergoes another transition from a spin-glass to a ferromagnetic phase. The control parameter that drives this transition is the degree of randomness (frustration) of the coupling constants between the photonic spins. Our results shed light on the ongoing effort of taming the flow of electromagnetic 
radiation in nonlinear multimode photonic networks. We expect that our approach can be applicable to a range of other nonlinear bosonic settings. We also expect to spark the interest of the statistical physics community since the mathematical models that are typically used to describe light transport in multimode systems fall outside the traditional spin-network framework.

\section{ACKNOWLEDGMENTS}

We acknowledge useful discussions with D. Arovas, G. Bunin, J. Feinberg, and D. Podolsky. We also acknowledge the assistance of S. Suwannarat with the preparation of Fig. 1. This research was supported by the ONR via Grant No. N00014-19-1-2480. B. S. acknowledges the hospitality of WTICS lab at Wesleyan University where this work was initiated.

Note added in proof.-Recently, the Rayleigh-Jeans statistics was derived in [76], using the grand canonical approach of Sec. II C.

[1] The Fermi-Pasta-Ulam Problem, Lecture Notes in Physics Vol. 728, edited by G. Gallavotti (Springer, Berlin, 2008).

[2] R. K. Pathria and P. D. Beale, Statistical Mechanics, 3rd ed. (Elsevier Science, Oxford, 2011).

[3] E. T. Jaynes, Information Theory and Statistical Mechanics, Phys. Rev. 106, 620 (1957).

[4] V. E. Zakharov, V. S. L'vov, and G. Falkovich, Kolmogorov Spectra of Turbolence I: Wave Turbulence (Springer Science \& Business Media, Berlin, 2012).

[5] A. C. Newell and B. Rumpf, Wave Turbulence, Annu. Rev. Fluid Mech. 43, 59 (2011).

[6] S. Trotzky, Y.-A. Chen, A. Flesch, I. P. McCulloch, U.Schollwöck, J. Eisert, and I. Bloch, Probing the Relaxation towards Equilibrium in an Isolated Strongly Correlated One-Dimensional Bose Gas, Nat. Phys. 8, 325 (2012).

[7] L. Pitaevskii and S. Stringari, Bose-Einstein Condensation (Oxford University Press, Oxford, 2003).

[8] N. Cherroret, T. Karpiuk, B. Grémaud, and C. Miniatura, Thermalization of Matter Waves in Speckle Potentials, Phys. Rev. A 92, 063614 (2015); N. Śantić, A. Fusaro, S. Salem, J. Garnier, A. Picozzi, and R. Kaiser, Nonequilibrium Precondensation of Classical Waves in Two Dimensions Propagating through Atomic Vapors, Phys. Rev. Lett. 120, 055301 (2018).

[9] Y. Silberberg, Y. Lahini, Y. Bromberg, E. Small, and R. Marandotti, Universal Correlations in a Nonlinear Periodic ID System, Phys. Rev. Lett. 102, 233904 (2009).

[10] J. Klaers, J. Schmitt, F. Vewinger, and M. Weitz, BoseEinstein Condensation of Photons in an Optical Microcavity, Nature (London) 468, 545 (2010).

[11] E. Small, R. Pugatch, and Y. Silberberg, BerezinskiiKosterlitz-Thouless Crossover in a Photonic Lattice, Phys. Rev. A 83, 013806 (2011).
[12] C. Sun, S. Jia, C. Barsi, S. Rica, A. Picozzi, and J. W. Fleischer, Observation of the Kinetic Condensation of Classical Waves, Nat. Phys. 8, 470 (2012).

[13] G. Situ, S. Muenzel, and J. Fleischer, Berezinskii-KosterlitzThouless Transition in a Photonic Lattice, arXiv:1304.6980.

[14] W. H. Renninger and F. W. Wise, Optical Solitons in Graded-Index Multimode Fibres, Nat. Commun. 4, 1719 (2013).

[15] A. Picozzi, J. Garnier, T. Hansson, P. Suret, S. Randoux, G. Millot, and D. N. Christodoulides, Optical Wave Turbulence: Towards a Unified Nonequilibrium Thermodynamic Formulation of Statistical Nonlinear Optics, Phys. Rep. 542, 1 (2014).

[16] L. G. Wright, D. N. Christodoulides, and F. W. Wise, Controllable Spatiotemporal Nonlinear Effects in Multimode Fibres, Nat. Photonics 9, 306 (2015).

[17] L. G. Wright, D. N. Christodoulides, and F. W. Wise, Spatiotemporal Mode-Locking in Multimode Fiber Lasers, Science 358, 94 (2017).

[18] K. Krupa, A. Tonello, B. Shalaby, M. Fabert, A. Barthélémy, G. Millot, S. Wabnitz, and V. Couderc, Spatial Beam Self-Cleaning in Multimode Fibres, Nat. Photonics 11, 237 (2017).

[19] F. Wu, A. Hassan, and D. Christodoulides, Thermodynamic Theory of Highly Multimoded Nonlinear Optical System, Nat. Photonics 13, 776 (2019).

[20] C. Barsi, W. Wan, and J. W. Fleischer, Imaging through Nonlinear Media via Digital Holography, Nat. Photonics 3, 211215 (2019).

[21] M. Plöschner, T. Tyc, and T. Cizmár, Seeing through Chaos in Multimode Fibres, Nat. Photonics 9, 529 (2015).

[22] A. P. Mosk, A. Lagendijk, G. Lerosey, and M. Fink, Controlling Waves in Space and Time for Imaging and Focusing in Complex Media, Nat. Photonics 6, 283 (2012).

[23] D. J. Richardson, J. M. Fini, and L. E. Nelson, Space Division Multiplexing in Optical Fibers, Nat. Photonics 7, 354 (2013).

[24] S. Fan and J. M. Kahn, Principal Modes in Multimode Waveguides, Opt. Lett. 30, 135 (2005).

[25] K.-P. Ho and J. M. Kahn, Mode Coupling and Its Impact on Spatially Multiplexed Xystems, Optical Fiber Telecommunications VIB (Elsevier, New York, 2013).

[26] M. Parto, F. Wu, P. Jung, K. Makris, and D. Christodoulides, Thermodynamic Conditions Governing the Optical Temperature and Chemical Potential in Nonlinear Highly Multimoded Photonic Systems, Opt. Lett. 44, 3936 (2019).

[27] P. Buonsante, R. Franzosi, and A. Smerzi, Phase Transitions at High Energy Vindicate Negative Microcanonical Temperature, Phys. Rev. E 95, 052135 (2017).

[28] T. Kottos and B. Shapiro, Thermalization of Strongly Disordered Nonlinear Chains, Phys. Rev. B 83, 062103 (2011).

[29] P. Aschieri, J. Garnier, C. Michel, V. Doya, and A. Picozzi, Condensation and Thermalization of Classical Optical Waves in a Waveguide, Phys. Rev. A 83, 033838 (2011).

[30] S. Dyachenko, A. Newell, A. Pushkarev, and V. Zakharov, Optical Turbulence: Weak Turbulence, Condensates and Collapsing Filaments in the Nonlinear Schrödinger Equation, Physica (Amsterdam) 57D, 96 (1992). 
[31] A. Picozzi, Towards a Nonequilibrium Thermodynamic Description of Incoherent Nonlinear Optics, Opt. Express 15, 9063 (2007).

[32] A. Picozzi and S. Rica, Condensation of Classical Optical Waves Beyond the Cubic Nonlinear Schrödinger Equation, Opt. Commun. 285, 5440 (2012).

[33] G. Lopez-Galmiche, Z. Eznaveh, M. Eftekhar, J. Antonio Lopez, L. Wright, F. Wise, D. Christodoulides, and R. Amezcua Correa, Visible Supercontinuum Generation in a Graded Index Multimode Fiber Pumped at 1064 nm, Opt. Lett. 41, 2553 (2016).

[34] Z. Liu, L. Wright, D. Christodoulides, and F. Wise, Kerr Self-Cleaning of Femtosecond-Pulsed Beams in GradedIndex Multimode Fiber, Opt. Lett. 41, 3675 (2016).

[35] A. Niang, T. Mansuryan, K. Krupa, A. Tonello, M. Fabert, P. Leproux, D. Modotto, O. N. Egorova, A. E. Levchenko, D. S. Lipatov, S. L. Semjonov, G. Millot, V. Couderc, and S. Wabnitz, Spatial Beam Self-Cleaning and Supercontinuum Generation with Yb-Doped Multimode Graded-Index Fiber Taper Based on Accelerating Self-Imaging and Dissipative Landscape, Opt. Express 27, 24018 (2019).

[36] E. V. Podivilov, D. S. Kharenko, V. A. Gonta, K. Krupa, O. S. Sidelnikov, S. Turitsyn, M. P. Fedoruk, S. A. Babin, and S. Wabnitz, Hydrodynamic 2D Turbulence and Spatial Beam Condensation in Multimode Optical Fibers, Phys. Rev. Lett. 122, 103902 (2019).

[37] A. Fusaro, J. Garnier, K. Krupa, G. Millot, and A. Picozzi, Dramatic Acceleration of Wave Condensation Mediated by Disorder in Multimode Fibers, Phys. Rev. Lett. 122, 123902 (2019).

[38] H. E. Stanley, Introduction to Phase Transition and Critical Phenomena (Oxford University Press, New York, 1971).

[39] C. Domb and J. Lebowitz, Phase Transitions and Critical Phenomena (Academic Press, London, 2000).

[40] S. Friedli and Y. Velenik, Statistical Mechanics of Lattice Systems (Cambridge University Press, Cambridge, England, 2017).

[41] J. Garnier, A. Fusaro, K. Baudin, C. Michel, K. Krupa, G. Millot, and A. Picozzi, Wave Condensation with Weak Disorder versus Beam Self-Cleaning in Multimode Fibers, Phys. Rev. Lett. 100, 053835 (2019).

[42] L. Leuzzi, C. Conti, V. Folli, L. Angelani, and G. Ruocco, Phase Diagram and Complexity of Mode-Locked Lasers: From Order to Disorder, Phys. Rev. Lett. 102, 083901 (2009).

[43] C. Conti and L. Leuzzi, Complexity of Waves in Nonlinear Disordered Media, Phys. Rev. B 83, 134204 (2011).

[44] M. Nixon, R. Ronen, A. A. Friesem, and N. Davidson, Observing Geometric Frustration with Thousands of Coupled Lasers, Phys. Rev. Lett. 110, 184102 (2013).

[45] F. Antenucci, C. Conti, A. Crisanti, and L. Leuzzi, General Phase Diagram of Multimodal Ordered and Disordered Lasers in Closed and Open Cavities, Phys. Rev. Lett. 114, 043901 (2015).

[46] D. Pierangeli, A. Tavani, F. Di Mei, A. J. Agranat, C. Conti, and E. DelRe, Observation of Replica Symmetry Breaking in Disordered Nonlinear Wave Propagation, Nat. Commun. 8 , 1501 (2017).
[47] M. Parto, W. Hayenga, A. Marandi, D. N. Christodoulides, and M. Khajavikhan, Realizimg Spin-Hamiltonians in Nanoscale Active Photonic Lattices, arXiv:1912.12075.

[48] K. J. Vahala, Optical Microcavities, Nature (London) 424, 839 (2003).

[49] S. K. Turitsyn, B. G. Bale, and M. P. Fedoruk, DispersionManaged Solitons in Fibre Systems and Lasers, Phys. Rep. 521, 135 (2012).

[50] Y. S. Kivshar, T. J. Alexander, and S. K. Turitsyn, Nonlinear Modes of a Macriscopic Quantum Oscillator, Phys. Lett. A 278, 225 (2001).

[51] P. Chiarawongse, H. Li, W. Xiong, C. W. Hsu, H. Cao, and T. Kottos, Statistical Description of Transport in Multimode Fibers with Mode-Dependent Loss, New J. Phys. 20, 113028 (2018).

[52] Y. Li, D. Cohen, and T. Kottos, Coherent Wave Propagation in Multimode Systems with Correlated Noise, Phys. Rev. Lett. 122, 153903 (2019).

[53] Y. Li, D. Cohen, and T. Kottos, Anomalous Decay of Coherent Wave Propagation in Multi-Mode Fibers (to be published).

[54] K. M. Frahm and D. L. Shepelyansky, Emergence of Quantum Ergodicity in Rough Billiards, Phys. Rev. Lett. 79, 1833 (1997).

[55] A. Barnett, D. Cohen, and E. J. Heller, Deformations and Dilations of Chaotic Billiards: Dissipation Rate, and Quasiorthogonality of the Boundary Wave Functions, Phys. Rev. Lett. 85, 1412 (2000).

[56] A. Barnett, D. Cohen, and E. J. Heller, Rate of Energy Absorption for a Driven Chaotic Cavity, J. Phys. A 34, 413 (2001).

[57] Y. Hlushchuk, L. Sirko, U. Kuhl, M. Barth, and H.-J. Stöckmann, Experimental Investigation of a Regime of Wigner Ergodicity in Microwave Rough Billiards, Phys. Rev. E 63, 046208 (2001).

[58] K. Rasmussen, T. Cretegny, P. Kevrekidis, and N. Grønbech-Jensen, Statistical Mechanics of a Discrete Nonlinear System, Phys. Rev. Lett. 84, 3740 (2000).

[59] M. Johansson and K. Rasmussen, Statistical Mechanics of General Discrete Nonlinear Schrödinger Models: Localization Transition and Its Relevance for Klein-Gordon Lattices, Phys. Rev. E 70, 066610 (2004).

[60] B. Rumpf, Transition Behavior of the Discrete Nonlinear Schrödinger Equation, Phys. Rev. E 77, 036606 (2008).

[61] U. Levy and Y. Silberberg, Equilibrium Temperatures of Discrete Nonlinear Systems, Phys. Rev. B 98, 060303(R) (2018).

[62] S. Flach and A. Gorbach, Discrete Breathers-Advances in Theory and Applications, Phys. Rep. 467, 1 (2008).

[63] In the work of L. Ermann and D. Shepelyansky, Quantum Gibbs Distribution from Dynamical Thermalization in Classical Nonlinear Lattices, New J. Phys. 15, 123004 (2013) (and references therein), the set $\left|C_{\alpha}\right|^{2}$ was normalized to unity and identified with the occupation probabilities of the modes. This assumption led, of course, to a different expression for the entropy and to results in contradiction with the present work and with Refs. [19,26,27,29-31]

[64] L. D. Landau and E. M. Lifshitz, Theoretical Physics: Statistical Physics (Butterworth-Heinemann, London, 1980), Vol. 5. 
[65] H.-J. Stöckmann, Quantum Chaos: An Introduction (Cambridge University Press, Cambridge, England, 2007).

[66] S. Friedli and Y. Velenik, Statistical Mechanics of Lattice Systems (Cambridge University Press, Cambridge, England, 2017).

[67] D. Sherrington and S. Kirkpatrick, Solvable Model of a Spin-Glass, Phys. Rev. Lett. 35, 1792 (1975).

[68] S. F. Edwards and P. W. Anderson, Theory of Spin Glasses, J. Phys. F5, 965 (1975).

[69] C. Skokos, E. Gerlach, J Bodyfelt, G. Papamikos, and S. Eggl, High Order Three Part Split Symplectic Integrators: Efficient Techniques for the Long Time Simulation of the Disordered Discrete Nonlinear Schrödinger Equation, Phys. Lett. A 378, 1809 (2014).

[70] E. Gerlach, J. Meichsner, and C. Skokos, On the Symplectic Integration of the Discrete Nonlinear Schrodinger Equation with Disorder, Eur. Phys J. Special Topics 225, 1103 (2016).
[71] P. J. Channell and F. R. Neri, An Introduction to Symplectic Integrators, Field Inst. Commun. 10, 45 (1996).

[72] K. H. Fischer and J. A. Hertz, Spin Glasses (Cambridge University Press, Cambridge, England, 1991).

[73] M. Mezard, G. Parisi, and M. A. Virasoro, Spin Glass Theory and Beyond, (World Scientific, Singapore, 1987), ISBN 978-9971-5-0115-0.

[74] A transition, as well as negative temperatures (inverted population), can only happen if the total energy is allowed to become nonextensive.

[75] A detailed analysis of the correlation length and of the crossover between various spin-phases is currently under investigation and will be published elsewhere.

[76] K. G. Makris, F. O. Wu, P. S. Jung, and D. N. Christodoulides, Statistical Mechanics of Weakly Nonlinear Optical Multimode Gases, Opt. Lett. 45, 1651 (2020) 